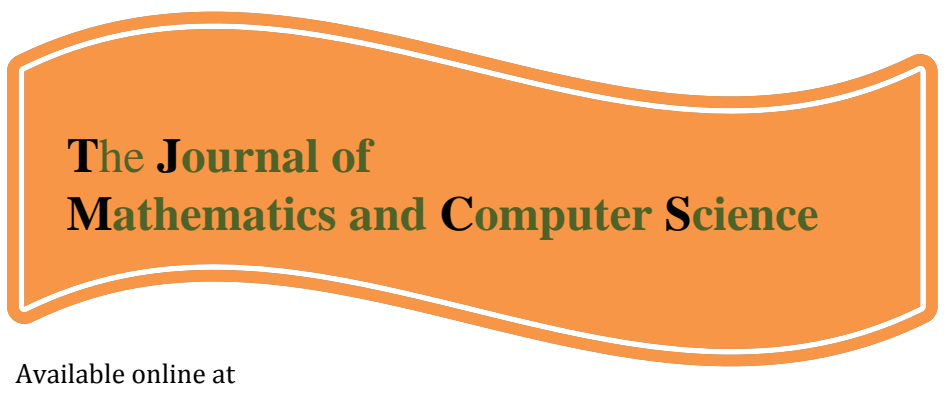

http://www.TIMCS.com

The Journal of Mathematics and Computer Science Vol .2 No.3 (2011) 483-487

\title{
The uniqueness theorem for boundary value problem with aftereffect and eigenvalue in the boundary condition*
}

\author{
A. H. dabbaghian' ${ }^{1}$, S. Akbarpoor ${ }^{2}$, J. Vahidi ${ }^{3}$ \\ ${ }^{1}$ Islamic Azad University, Neka Branch, a.dabbaghian@math.com \\ ${ }^{2}$ Islamic Azad University, Jouybar Branch \\ 3Iran University of Science and Technology, j.vahidi@b-iust.ac.ir
}

Received: September 2010, Revised: December 2010

Online Publication: January 2011

\begin{abstract}
In this paper, we consider the boundary value problem with eigenvalue in the boundary condition. We obtain an asymptotic formula for nodal points. In addition, we give the uniquess theorem from nodes of its eigenfunctions.
\end{abstract}

Keywords: nodal points, uniqueness theorem, eigenvalues.

\section{Introduction}

Inverse nodal problems consist in recovering operators from given nodes(zeros) of their eigenfunctions. Mclaughlin seems to have been the first to consider this sort of inverse problem for the one-dimensional Schrodinger equations on an interval with Dirichlet boundary conditions.(see[3]). We consider boundary value problem with"aftereffect" on a finite interval and with eigenvalue in the boundary condition:

\footnotetext{
* This paper mainly based on "On the determination of system of differential equations with singularities and turning points" which supported by Islamic Azad University, Neka Branch.
} 
$l y(x):=-y^{\prime \prime}(x)+q(x) y(x)+\int_{0}^{x} M(x-t) y(t) d t=\lambda^{2} y(x), \quad \circ<x<\pi$,

With the boundary conditions

$y^{\prime}(\circ, \lambda)-\lambda y(\circ, \lambda)=\circ$

$y^{\prime}(\pi, \lambda)+H y(\pi, \lambda)=\circ$.

Here $\lambda$ is the spectral parameter; $q(x) \in L_{2}(\circ, \pi)$ and $H$ are real parameter. The presence of an aftereffect in a mathematical model produces qualitative changes in the study of the inverse problem. The uniqueness theorem for boundary value problem with aftereffect by the transformation operator method was studied in [1]. It says that the function $M(x)$ is uniquely determined from the given $q(x)$ and the spectrum $\left\{\lambda_{n}\right\}_{n \geq 1}$.

Such, we denote that in [2] inverse nodal problem for the Sturm-Liouville problem $y^{\prime \prime}+\left[\lambda^{2}+\mu-q(x)\right] y=\circ$ was studied with boundary conditions (2)-(3). In thispaper, using of the nodal points we show uniqueness of $M(x), H$. In other word, the function $M(x)$ and $H$ are uniquely determined from a dense set of nodal points and given $q(x)$. The paper is organized as follows. In section 2 , we obtain an asymptotic of the nodal points and will give a uniqueness theorem. In section 3 , we prove the uniqueness theorem for the solution of this inverse problem.

\section{Asymptotic of the nodal points}

Let $y(x, \lambda)$ be solution of (1) under the initial conditions $y(\circ, \lambda)=1, y^{\prime}(\circ, \lambda)=\lambda$. For $|\lambda| \rightarrow \infty$, the following asymptotic formula hold

$$
y(x, \lambda)=\cos \lambda x+\sin \lambda x+o(1)
$$

uniformly with to $x \in[\circ, \pi]$. The funcyion $y(x, \lambda)$ is the solution of the integral equation

$$
\begin{aligned}
& y(x, \lambda)=\cos \lambda x+\sin \lambda x \\
& +\int_{0}^{x} \frac{\sin \lambda(x-t)}{\lambda}\left(q(t) y(t, \lambda)+\int_{0}^{t} M(t-s) y(s, \lambda) d s\right) d t .
\end{aligned}
$$

Further

$$
\begin{aligned}
& y^{\prime}(x, \lambda)=-\lambda \sin \lambda x+\lambda \cos \lambda x \\
& +\int_{0}^{x} \cos \lambda(x-t)\left(q(t) y(t, \lambda)=+\int_{0}^{t} M(t-s) y(s, \lambda) d s\right) d t .
\end{aligned}
$$

Substituting the asymptotic for $y(x, \lambda)$ from (4) into the right-hand sides of (5), we calculate

$$
\begin{aligned}
& y(x, \lambda)=\cos \lambda x+\sin \lambda x+\frac{\sin \lambda x-\cos \lambda x}{2 \lambda} \int_{0}^{x} q(t) d t \\
& +\frac{1}{\lambda} \int_{0}^{x} \sin \lambda(x-t) \int_{0}^{t} M(t-s)[\cos \lambda s+\sin \lambda s] d s d t+o\left(\frac{1}{\lambda}\right)
\end{aligned}
$$


And

$$
\begin{aligned}
& y^{\prime}(x, \lambda)=\lambda(\cos \lambda x-\sin \lambda x)+\frac{\sin \lambda x+\cos \lambda x}{2} \int_{0}^{x} q(t) d t \\
& +\int_{0}^{x} \cos \lambda(x-t) \int_{0}^{t} M(t-s)[\cos \lambda s+\sin \lambda s] d s d t+o(1) .
\end{aligned}
$$

Denote

$$
\Delta\left(\lambda_{n}\right)=y^{\prime}(\pi, \lambda)+H y(\pi, \lambda)
$$

Further

$$
\Delta\left(\lambda_{n}\right)=(H+\lambda) \cos \lambda \pi+(H-\lambda) \sin \lambda \pi+\frac{\sin \lambda \pi+\cos \lambda \pi}{2} \int_{0}^{\pi} q(t) d t+\kappa(\lambda),
$$

Where

$$
\kappa(\lambda)=\int_{0}^{\pi} \cos \lambda(\pi-t) \int_{0}^{t} M(t-s)[\cos \lambda s+\sin \lambda s] d s d t+o(1)
$$

By the well-known method (see, for example [1]) using (10) and Rouche's theorem one can prove that $L$ has infinitely many eigenvalues $\lambda_{n}, n \in Z-\{\circ\}$, of the form

$$
\lambda_{n}=n+\frac{1}{\pi}+\frac{H}{n \pi}+\frac{1}{2 n \pi} \int_{0}^{\pi} q(t) d t+\frac{\kappa_{n}}{n}, \quad\left\{\kappa_{n}\right\} \in l_{2} .
$$

The eigenfunctions of the boundary value problem have the form $y_{n}(x)=y\left(x, \lambda_{n}\right)$. Substituting (11) into (7) we obtain the following asymptotic formulae for $n \rightarrow \infty$ uniformly in $x$ :

$$
\begin{aligned}
& y_{n}(x)=1+\frac{1}{2 n}=\sin \left(n+\frac{1}{\pi}\right) x+\left(\frac{H}{n \pi}+\frac{1}{2 n \pi} \int_{0}^{\pi} q(t) d t\right) x \\
& -\frac{1}{n} \int_{0}^{x} \sin \left(n+\frac{1}{\pi}\right) t \int_{0}^{t} M(t-s)\left[1+\sin \left(n+\frac{1}{\pi}\right) s\right] d s d t+o\left(\frac{1}{n}\right) .
\end{aligned}
$$

For the boundary value problem an analog of Sturm's oscillation theorem is true. More precisely, the eigenfunction $y_{n}(x)$ has exactly $n$ zeros inside the interval $(\circ, \pi)$. Namely:

$\circ<x_{n}^{1}<x_{n}^{2}<\ldots<x_{n}^{j}<\pi, \quad j=1,2, \ldots, n-1$.

The set $X=\left\{x_{n}^{j}\right\}_{n \geq 1, j=1, n}$ is called the set of nodal points of the boundary value problem. It is shown that the set of all nodal points $\left\{x_{n}^{j}\right\}$ is dense in $[\circ, \pi]$. By (12) we obtain the following asymptotic formulae for nodal points as $n \rightarrow \infty$ uniformly in $j$ :

$$
\begin{aligned}
& x_{n}^{j}=\frac{j \pi-1}{n+\frac{1}{\pi}}+\frac{1}{n+\frac{1}{\pi}}\left[-\frac{1}{2 n}-\frac{1}{n \pi}\left(H+\frac{1}{2} \int_{0}^{\pi} q(t) d t\right) x\right. \\
& \left.+\frac{1}{n} \int_{0}^{x} \sin \left(n+\frac{1}{\pi}\right) t \int_{0}^{t} M(t-s)\left[1+\sin \left(n+\frac{1}{\pi}\right) s\right] d s d t\right]+o\left(\frac{1}{n^{2}}\right) .
\end{aligned}
$$


Now, we will give a uniqueness theorem. It says that the function $M(x), H$ are uniquely determined by a dense subset of the nodes and the function $q(x)$.

\section{Uniqueness theorem by using nodal points}

Theorem1 Fix $x \in[\circ, \pi]$. Let $\left\{x_{n}^{j}\right\} \in X$ be chosen such that

$$
\lim _{n \rightarrow \infty} x_{n}^{j_{n}}=x .
$$

Then there exists a finite limit

$$
g(x):=\lim _{n \rightarrow \infty} n\left[\left(n+\frac{1}{\pi}\right) x_{n}^{j}-j \pi+1\right]
$$

So that

$$
g(x)=-\frac{1}{2}-\frac{1}{\pi}\left(H+\frac{1}{2} \int_{0}^{\pi} q(t) d t\right) x
$$

That $H$ can be constructed vie the formulae :

$$
H=-g(\pi)-\frac{1}{2}\left(1+\int_{0}^{\pi} q(t) d t\right)
$$

Theorem 2 Let $q(x)=\widetilde{q}(x)$ on $[\circ, \pi]$ then the function $M$ and $H$ are uniquely determined by any dense subset of the nodes in $[\circ, \pi]$.

Proof. Consider the following equation

$$
\tilde{l} y(x):=-\tilde{y}^{\prime \prime}(x)+q(x) \tilde{y}(x)+\int_{0}^{x} \tilde{M}(x-t) \tilde{y}(t) d t=\tilde{\lambda}^{2} y(x), \quad \circ<x<\pi,
$$

Where $\tilde{M}$ have the same properties of $M$. Let $x_{n}^{j}=\tilde{x}_{n}^{j}$. If we multiply (1) by $\tilde{y}$ and (17) by $y$, and then subtract, we obtain

$$
\left[\tilde{y}(x) y^{\prime}(x)-\tilde{y}^{\prime}(x) y(x)\right]^{\prime}+\int_{0}^{x}[\tilde{M}(x-t) y(x) \tilde{y}(t)-M(x-t) y(t) \tilde{y}(x)] d t=\left(\tilde{\lambda}_{n}^{2}-\lambda_{n}^{2}\right) y \tilde{y}
$$

Integrating (18) from $x_{n}^{j}$ to $\pi$, we obtain

$$
\begin{aligned}
& {\left[\tilde{y}_{n}(\pi) y_{n}^{\prime}(\pi)-\tilde{y}_{n}^{\prime}(\pi) y_{n}(\pi)\right]+\int_{x_{n}^{j}}^{\pi} \int_{0}^{x}[\tilde{M}(x-t) y(x) \tilde{y}(t)-M(x-t) y(t) \tilde{y}(x)] d t d x} \\
& =\int_{x_{n}^{j}}^{\pi}\left(\tilde{\lambda}_{n}^{2}-\lambda_{n}^{2}\right) y \tilde{y} d x . \\
& (\tilde{H}-H) \tilde{y}_{n}(\pi) y_{n}(\pi)=\int_{x_{n}^{j}}^{x} \int_{0}^{x}[M(x-t) y(t) \tilde{y}(x)-\tilde{M}(x-t) y(x) \tilde{y}(t)] d t d x \\
& +\int_{x_{n}^{j}}^{\pi}\left(\tilde{\lambda}_{n}^{2}-\lambda_{n}^{2}\right) y \tilde{y} d x .
\end{aligned}
$$

From the asymptotic forms of $y$ and $\tilde{y}$, we can obtain that $\left|y\left(\pi, \lambda_{n}\right) \tilde{y}\left(\pi, \lambda_{n}\right)\right|$ is bounded away from zero. Finally, if we take a sequence $x_{n}^{j}$ accumulating at $\pi$, the 
right-hand side tends to zero. Then $H=\tilde{H}$. Integrating (18) from $\circ$ to $x_{n}^{j}$, we obtain

$\int_{0}^{x_{2}^{j}} \int_{0}^{x}[\tilde{M}(x-t) y(x) \tilde{y}(t)-M(x-t) y(t) \tilde{y}(x)] d t d x=0$.

Further

$\int_{0}^{x_{0}^{j}} \int_{0}^{x}[\tilde{M}(x-t)-M(x-t)] \cos \lambda(x-t) d t d x$

$+\int_{0}^{x_{n}^{j}} \int_{0}^{x}[\tilde{M}(x-t)-M(x-t)] \sin \lambda(x+t) d t d x=0$

We take a sequence $x_{n}^{j}$ accumulating at an arbitrary $b \in[\circ, \pi]$. Hence,

$\int_{0}^{b} \int_{0}^{x}[\tilde{M}(x-t)-M(x-t)] \cos \lambda(x-t) d t d x$

$+\int_{0}^{b} \int_{0}^{x}[\tilde{M}(x-t)-M(x-t)] \sin \lambda(x+t) d t d x=0$.

On the other hand, from the completeness of the functions sin and cos we can conclude that $M$ is uniquely determined on $[\circ, \pi]$.

\section{References}

[1] G. Freiling, V.A. Yurko, Inverse Sturm-Liouville problems and their applications, NOVA scince publishers, New York, 2001.

[2] H. Koyunbakan, Inverse nodal problem differential operator with eigenvalue in the boundary condition, applied Mathematics Letters 21(2008)1301-1305.

[3] J.R. Mclaughlin, Inverse spectral theory using nodal points as data-a uniqueness result, J. Differential Equations 73(2)(1988)354-362. 